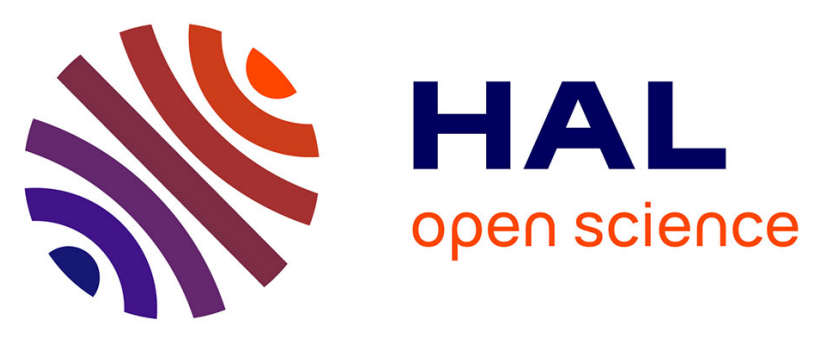

\title{
Vortex Induced Vibrations Analysis of a Cantilevered Blunt Plate by Proper Orthogonal Decomposition of TR-PIV and Structural Modal Analysis
}

Yann Watine, Céline Gabillet, Jacques-André Astolfi, Laetitia Pernod, Boris Lossouarn, Jean-François Deü

\section{To cite this version:}

Yann Watine, Céline Gabillet, Jacques-André Astolfi, Laetitia Pernod, Boris Lossouarn, et al.. Vortex Induced Vibrations Analysis of a Cantilevered Blunt Plate by Proper Orthogonal Decomposition of TR-PIV and Structural Modal Analysis. ASME 2020 Fluids Engineering Division Summer Meeting, FEDSM 2020, Jul 2020, Virtual conference, United States. 10.1115/FEDSM2020-20226 . hal-03196687

\section{HAL Id: hal-03196687 \\ https://hal.science/hal-03196687}

Submitted on 21 Dec 2021

HAL is a multi-disciplinary open access archive for the deposit and dissemination of scientific research documents, whether they are published or not. The documents may come from teaching and research institutions in France or abroad, or from public or private research centers.
L'archive ouverte pluridisciplinaire HAL, est destinée au dépôt et à la diffusion de documents scientifiques de niveau recherche, publiés ou non, émanant des établissements d'enseignement et de recherche français ou étrangers, des laboratoires publics ou privés. 


\title{
VORTEX INDUCED VIBRATIONS ANALYSIS OF A CANTILEVERED BLUNT PLATE BY PROPER ORTHOGONAL DECOMPOSITION OF TR-PIV AND STRUCTURAL MODAL ANALYSIS
}

\author{
Yann Watine ${ }^{1}$, Céline Gabillet ${ }^{1}$, Jacques-André Astolfi ${ }^{1}$, Laetitia Pernod ${ }^{2}$, Boris Lossouarn ${ }^{2}$, \\ Jean-François Deü ${ }^{2}$
}

\author{
${ }^{1}$ IRENav, Naval Academy Research Institute, BCRM Brest - École Navale - CC600 \\ 29240, Brest, Cedex 9, France \\ ${ }^{2}$ Laboratoire de Mécanique des Structures et des Systèmes Couplés (LMSSC) \\ Conservatoire national des arts et métiers (Cnam) \\ 292 rue Saint-Martin, 75 003, Paris, France
}

\begin{abstract}
The present work focuses on the experimental characterization of the vortex shedding and on the induced vibrations of a cantilevered blunt rectangular aluminum plate of chord to thickness ratio 16 , immersed in a uniform water flow in the hydrodynamic tunnel of the French Naval Academy Research Institute. Experiences have been conducted for Reynolds numbers Re (based on chord length) ranging from 2.5 $\times 10^{5}$ to $10.5 \times 10^{5}$ at zero degrees incidence. Special attention has been paid to the interaction of the structural response and the flow dynamics at the twisting resonance. For this purpose, wake structures have been analyzed by Time Resolved Particle Image Velocimetry (TR-PIV) and the structural response of the plate has been examined by laser vibrometry. The von Karman vortex street has been characterized by statistical analysis and Proper Orthogonal Decomposition of PIV velocity fields and the structure is analyzed through modal analysis. The nearwake's structure has been examined for three different Reynolds numbers: (i) at $\mathrm{Re}=3.0 \times 10^{5}$, corresponding to vortex induced structural response at constant Strouhal number; (ii) at $\mathrm{Re}=4.5 \times 10^{5}$, corresponding to mechanical resonance but dissociated vortex shedding and (iii) at $\mathrm{Re}=5.4 \times 10^{5}$, corresponding to lock-in of the vortex shedding at the mechanical resonance. At $\mathrm{Re}=4.5 \times 10^{5}$, at mechanical resonance, it reveals the occurrence of an energy transfer between the shear layer and the bubble wake vortex which cancels synchronization of the structural vibration with the vonKarman vortex street.
\end{abstract}

Keywords: Vortex-induced vibrations, modal decomposition, TR-PIV. POD, Modal Analysis



\section{INTRODUCTION}

Immersed structures such as hydrofoils, rudders or propellers are highly subjected to flow induced vibrations [1]. These vibrations lead to disturbing noise and can even induce structural fatigue. The development of immersed lifting surfaces for fast sailing yachts has placed the flow induced vibrations issues as a major concern during the design process. Flow induced vibrations are at the origin of acoustic noise generated by ship propellers which is a major issue for the discretion of naval ships. Usually, marine propellers are designed by considering mainly the hydrodynamic aspect and a less attention is paid on the deformations and vibrations of the structure interacting with the flow. In some extreme cases, the flow excitation can tune in with the structural response and 
cause dramatic deterioration of the structure as well as strong noise emission. On the other hand, understanding the origin of flow induced vibrations will open the way to new processes for controlling the structural vibrations [2] and will increase the efficiency of marine structures.

One topic of great interest for naval applications is the coupling between trailing edge vortex shedding and structural response. It requires conducting studies to understand the physics of Fluid Structure Interaction of hydro-elastic structures with a certain degree of elasticity at moderate to large Reynolds numbers in a high density fluid. When considering the literature survey, few studies deal with flow induced vibration of elongated bodies. Much investigations of vortex induced vibrations (VIV) focused on elastically mounted rigid bodies in a cross flow, moving with different degrees of freedom at moderate Reynolds numbers [3] and there is still a need to explore higher Reynolds numbers effects. Many works referring on elongated bodies concerns the wake and forces analyses downstream pitching-heaving bodies for bio mimicry propulsion system as reported by Triantafyllou et al (1993) [4] and Moubogha et al. (2017) [5] .

In particular, most of the studies dedicated to flat plates, aimed at characterizing the vortex shedding but they didn't consider induced vibrations. They evidenced a discrepancy in the Strouhal number according to the chord to thickness ratio, the type of edge, the angle of attack and the Reynolds number [6]. The sensitivity of vortex shedding to the Reynolds number is enhanced at small angle of attack, at which flow reattachment occurs [7]. Hence, for an elongated blunt plate, vortices shedding from the leading edge can interact in a different way with the von Karman vortex street and the flow is considered as much more complex than flows on short blunt bodies [8].

The present paper deals with an original experiment to study the interactions between the near-wake of a cantilevered blunt aluminum plate immersed in a uniform water flow at zero degree of incidence and the structural vibrations of the plate. The near wake has been characterized by Time-Resolved PIV. The vibrations of the structure have been analyzed by the use of a Doppler laser vibrometer. The analysis is performed trough flow velocities evolution ranging between $2.5 \mathrm{~m} / \mathrm{s}$ to $10.5 \mathrm{~m} / \mathrm{s}$, corresponding to Reynolds numbers based on the chord length between $2.5 \times 10^{5}$ and $10.5 \times 10^{5}$. The near wake has been investigated in details for three values of the Reynolds number corresponding respectively to (i) no mechanical resonance, (ii) mechanical resonance, and (iii) mechanical resonance and lockin of the vortex shedding.

The paper is organized as follows: section 2 is a presentation of the experimental set-up and methods. The results are presented and discussed in section 3. In this section, the near wake and the structure response are analyzed through Proper Orthogonal Decomposition (POD) analysis and Modal Analysis respectively.

\section{MATERIALS AND METHODS}

The experiments were carried out in the hydrodynamic tunnel of the French Naval Academy Research Institute (IRENav). The test section is $192 \mathrm{~mm}$ squared and $1 \mathrm{~m}$ long (cf. Figure 1). At the entrance of the test section, the upstream velocity and pressure are regulated with an accuracy of $\pm 2 \%$ and $\pm 2.5 \%$ respectively. For the present study, the upstream flow velocity $U_{0}$ ranges between 2.5 and $10.5 \mathrm{~m} . \mathrm{s}^{-1}$. The pressure was adjusted at $\mathrm{P}=1013 \mathrm{~Pa}$. The free stream turbulence intensity in the middle of the test section is $2 \%$.



FIGURE 1: TEST SECTION OF THE HYDRODYNAMIC TUNNEL AT FRENCH NAVAL ACADEMY

A rectangular cantilever blunt aluminum plate of chord $C=100$ $\mathrm{mm}$, span $\mathrm{B}=191 \mathrm{~mm}$ and thickness $\mathrm{D}=6 \mathrm{~mm}$ has been used (cf. Figure 2). Two piezoelectric patches have been used as an excitation source for the determination of the plate's natural frequencies. The plate has been assembled at the root on a rigid steel cylinder with a specifically clamped device to be embedded in the hydrodynamic test section and to allow the passage of the electric cables. This assembly aims to ensure an approximate clamped condition at the foil root forming a cantilevered structure. The clamped condition is ensured at $50 \%$ of the chord from the leading edge. The plate is mounted in the test section with no incidence. The maximum uncertainty in the angle of attack is $0.1^{\circ}$. The trailing edge of the plate is chosen as the origin of the $x-y$ coordinates, $\mathrm{x}$ being the streamwise direction, and y being the upward vertical direction (cf. figure 1).



FIGURE 2: CANTILEVERED RECTANGULAR BLUNT PLATE 


\subsection{Vibration measurements}

The vibration measurements and modal analyses were performed with two Polytec ${ }^{\circledR}$ laser vibrometers. The first one measured the vibration at a reference point on the structure whereas the second one is a PSV-400 scanning vibrometer to measure the vibration from a user defined grid. The laser vibrometer can detect vibration velocities from $0.01 \mathrm{~m} . \mathrm{s}^{-1}$ up to $10 \mathrm{~m} \cdot \mathrm{s}^{-1}$ using a HeNe laser $(\lambda=633 \mathrm{~nm})$ based on Doppler Effect. It is equipped with two analog velocity decoder VD-04 and VD-06. The scanner is a high precision scan unit, using mobile mirrors with an angular resolution lower than $0.002^{\circ}$ and an angular stability lower than $0.01^{\circ}$ per hour. It allows us to perform modal analysis by measuring the vibration level on a user-defined grid over the structure surface. The cross-spectrum between the reference laser point and a scanned measured point is computed to preserve the phase at a given frequency. This allows us to get the mode shape associated to a natural frequency as shown on Figure 4 and 5 for the two first modes.

The spectra amplitude can be presented in two ways: $\mathrm{m} \cdot \mathrm{s}^{-1}$ and/or $\mathrm{dB}$. Conversion from $\mathrm{S}=\mathrm{m} \cdot \mathrm{s}^{-1}$ to $\mathrm{dB}$ is made using the following relationship: $\mathrm{dB}=20 \log (\mathrm{S} / \mathrm{Sref})$, with $\mathrm{Sref}=1 \mathrm{~m} \cdot \mathrm{s}^{-1}$. The power spectral density of the vibration velocity of the structure was computed by using a Welch transform from an average of about 50 spectra at each point with a resolution $\Delta \mathrm{f}=0.625 \mathrm{~Hz}$. The scan was applied on a regular grid. Mode shapes and natural frequencies were characterized in still air and in still water. This was made by analyzing the vibration response to a white noise excitation provided by the piezoelectric transducers. Figure 3 displays the spectra measured in still air and in still water at point A (cf. Figure 2). A very narrow banded peak is visible at $100 \mathrm{~Hz}$ in water: it is characteristic of an electrical perturbation. The main peaks represent the natural frequencies of the plate. For this study, the frequencies of the first two peaks $\left(f_{1}, f_{2}\right)$ are of prime importance because these will be used as a reference when analyzing the mechanical resonance phenomenon. The values of the frequencies characteristic of the first bending and twisting modes are reported in Table 1 and denoted as $f_{1}$ and $f_{2}$ respectively. As shown, there is a strong decrease of around $50 \%$ of the frequency in water due to added-mass: $f_{\text {air }} / f_{\text {water }}=2.3$ for the $1^{\text {st }}$ bending mode, and $f_{\text {air }} / f_{\text {water }}=1.7$ for the $1^{\text {st }}$ twisting mode. $f_{3}$ is the natural frequency of the second bending mode measured in air. Its value has been added in Table 1.

Non dimensional parameters are the Reynolds number $R e=$ $\frac{U_{0} C}{v}$, and the reduced velocity based on the natural frequency of first twisting mode: $R U=\frac{U_{0}}{f_{2} D} \cdot v$ is the kinematic viscosity of the water.



FIGURE 3: VIBRATION SPECTRUM OF THE PLATE IN STILL AIR AND STILL WATER

\begin{tabular}{|l|l|l|}
\hline & \multicolumn{1}{|c|}{ Still air } & \multicolumn{1}{c|}{ Still water } \\
\hline$f_{\boldsymbol{I}}$ & $60.9 \mathrm{~Hz}$ & $25.6 \mathrm{~Hz}$ \\
\hline$f_{2}$ & $308.1 \mathrm{~Hz}$ & $181.9 \mathrm{~Hz}$ \\
\hline$f_{3}$ & N.A & $240.9 \mathrm{~Hz}$ \\
\hline
\end{tabular}

TABLE 1: MEASURED BENDING MODE FREQUENCY $f_{1}$ AND TWISITING MODE FREQUENCY $\mathrm{f}_{2}$ IN STILL AIR AND STILL WATER.



FIGURE 4: BENDING MODE SHAPE CORRESPONDING TO $\mathrm{f}_{1}$ IN STILL WATER.



FIGURE 5: TWISTING MODE SHAPE CORRESPONDING TO $\mathrm{f}_{2}$ IN STILL WATER.

\subsection{TR - PIV measurements}

Time resolved Particle Image Velocimetry (TR-PIV) has been used for the characterization of the flow downstream the plate. A Nd:YLF high-frequency laser with output energy of $11.7 \mathrm{~mJ} /$ pulse, wavelength of $527 \mathrm{~nm}$, and flash duration of $158.439 \mathrm{~ns}$ (first frame), was used to illuminate the flow in the vertical plane, aligned with the symmetry plane of the tunnel. The flow field has been imaged, using a $8-$ bit $1280 \times$ 800 pixel $^{2}$ Phantom v611 high-speed camera with $20 \mu \mathrm{m}$ pixel size, fitted with a $100 \mathrm{~mm}$ Zeiss Makro Planar lens, at a sampling frequency varying between $1000 \mathrm{~Hz}$ and $1400 \mathrm{~Hz}$ (according to the flow's speed). Time between pulses $\Delta t_{\text {pulse }}$ was adjusted with respect to the upstream velocity: $\Delta t_{\text {pulse }}(\mu s)=$ 
$\frac{438}{U_{0}(\mathrm{~m} / \mathrm{s})}$. The viewing area had dimensions $67.2 \times 42 \mathrm{~mm}^{2}$ (ie:11.2 $\left.\mathrm{D}^{*} 7 \mathrm{D}\right)$ in the $x$ and $y$ directions respectively and was centred along the axis $y=0$, which enabled to inspect the wake flow developing downstream of the plate blunt trailing edge, from $\mathrm{x}=-1.2 \mathrm{D}$ to $\mathrm{x}=10 \mathrm{D}$, in its near wake.

Analysis has been conducted, based on 1000 pairs of images collected. Velocity vectors were calculated using Adaptive PIV method implemented in DynamicStudio, applying an universal outlier detection method to eliminate spurious vector data with a kernel of $5 \times 5$ pixel $^{2}$ The spatial resolution, corresponding to interrogation area's size was $16^{*} 16$ pixels (ie: $0.14 \mathrm{D} \times 0.14 \mathrm{D})$ in the $\mathrm{x}$ and $\mathrm{y}$ directions.

Let $u$ and $v$ be the instantaneous velocity components of the fluid measured in the stream-wise and up-ward directions respectively. The Proper Orthogonal Decomposition (POD) is a method to extract modes of a dynamic system in terms of their contribution to the total energy of the system. It provides a reduced representation of data spatial-time series based on the most energetic modes of the system. Unlike the dynamic mode decomposition method which evidences coherent structures in time and space, the POD decomposition method provides only coherent structures in space [9].

Hence, the POD decomposition has been applied on the velocity field, using the snapshot method [10]. Let us denote $\left(\Phi_{\mathrm{u}}{ }^{\mathrm{i}}, \Phi_{\mathrm{v}}{ }^{\mathrm{i}}\right)$ the components of the eigen vector (POD mode) of mode number $i$. The most energetic POD modes $(1<=\mathrm{i}<=5)$ have been extracted and compared between different flow conditions. Also, the evolution with respect to time of the POD expansion coefficients $a_{i}$ of these modes has been analysed, based on frequency spectrum calculation. In this study, $a_{i}(t)$ represents the temporal dependence between the transient velocity field and the POD mode $\mathrm{i}$.

Spurious velocity vectors, as well as velocity fluctuations induced by low energetic turbulent structures have been suppressed from the instantaneous velocity fields by reconstruction of the velocity field as a linear combination of the $\mathrm{n}$ first modes, for which the cumulative energy is at least $90 \%$ of the total energy. To compare the global features of the wake for different upstream velocities, a time-averaged analysis has been performed, based on the reconstructed velocity field: $\mathrm{U}$ and $\mathrm{V}$ refer to the stream-wise and upward velocity components of the mean flow. Also, to characterize the vortices, shedding in the wake, the vortex identification algorithm based on the $\Gamma_{2}$ core identification method developed by Graftieaux et al [11] has been applied on the POD modes of the von Karman street. It makes it possible to determine the circulation $\Gamma$ and the area of the vortices. An example of vortices identification obtained in the von Karman street of the plate's wake at $\mathrm{U}_{0}=4.5 \mathrm{~m} / \mathrm{s}$ is presented in figure 6 : points in the vortices cores as well as vortices centers are displayed in black and green colours respectively and superimposed onto the cartography of the vorticity of POD mode number 1 .



FIGURE 6: VORTEX DETECTION ON POD FIRST MODE AT $4.5 \mathrm{M} / \mathrm{S}$.

\section{RESULTS}

\subsection{Structure and near wake response according to the upstream velocity}

Vibrometry measurements have been performed to evaluate the vibratory response of the plate structure at different flow velocities. Reynolds numbers range from $2.5 \times 10^{5}$ to $10.5 \times 10^{5}$ by steps of $0.5 \times 10^{5}$. For Reynolds ranging from $4.5 \times 10^{5}$ to $6.0 \times 10^{5}$, a refined step of $0.1 \times 10^{5}$ has been used for higher resolution. Figure 7 reports the cartography of the power spectral density of the vibration velocity of the structure according to the Reynolds number $R e$ and the reduced frequency $\frac{f_{S}}{f_{2}}$. $f_{S}$ is the frequency of the structure at which the power spectral density is given. For $R e$ in the intervals $\left[2.5 \times 10^{5}-4.0 \times 10^{5}\right],\left[6.0 \times 10^{5}-6.5 \times 10^{5}\right]$ and $\left[9.0 \times 10^{5}-10.5 \times 10^{5}\right]$, this figure highlights a linear evolution with the Reynolds number of the frequency $f_{S \max }$ at which the power spectral density is maximum. In this case, $f_{S} \max$ corresponds to the vortex shedding frequency $f_{v s}$ and the vortex shedding acts as a tonal excitation source on the structure. This evolution is associated with a constant Strouhal number $S t=\frac{f_{v S D}}{U_{0}}$ close to 0.20

The mechanical resonance phenomenon at the first twisting mode frequency of the plate $\left(f_{S \max }=f_{2}\right)$ is observed in the $R e$ range $\left[4,5 \times 10^{5}-6,0 \times 10^{5}\right]$ and the maximum value of the power spectral density is reached at Reynolds $5,7 \times 10^{5}$. For these conditions, the vibration amplitude of the plate is maximal. At the mechanical resonance, other maxima of the power spectral density are observed at harmonics of the natural frequency. Same features can be highlighted at the mechanical resonance of the second bending mode $\left(\frac{f_{S \max }}{f_{2}}=\frac{f_{3}}{f_{2}}=1.3\right)$. Note that the 
mechanical resonance of the first bending mode occurs out of the range of the $R e$ numbers of the study, at $R e<2.5 \times 10^{5}$.

Hereafter, we focus on the second twisting mode, interactions between vibrations and the flow.



FIGURE 7: CARTOGRAPHY OF THE POWER SPECTRAL DENSITY OF THE STRUCTURE RESPONSE. TOPOGRAPHIC REPRESENTATION.

In order to study the excitation source acting on the structure, the PIV dataset has been used to measure the characteristic frequency of the flow in the wake of the structure. The time-variations of the vertical fluctuating velocity component v', measured at a point located downstream the trailing edge at $\mathrm{x} / \mathrm{D}=3.3$ and aligned with the lower corner of the trailing edge at $y / D=-1$, have been analyzed by using a Fast Fourier Transform algorithm. The main wake frequency $f_{w}$, characteristic of the maxima of the power spectral density of v', has been evaluated. As a first approximation, $f_{w}$ is representative of the vortex shedding frequency. It is of prime interest to compare this frequency with the main structural response frequency $f_{S \text { max }}$, measured by vibrometry. Figure 8 reports the evolution of $f_{S \max }$ and $\mathrm{f}_{\mathrm{w}}$ as a function of Reynolds. The root mean square of the vibration velocity of the structure $\mathrm{V}_{S}{ }^{\text {rms }}$ and the maximal amplitude of the structural vibration velocity's spectrum $\mathrm{V}_{\mathrm{S}}{ }^{\max }$, measured at $f_{S \max }$, have also been reported on figure 8 . The root mean square is representative of the energy of the whole vibration velocity signal.



FIGURE 8: EVOLUTION WITH THE RE NUMBER OF THE STRUCTURAL VIBRATION VELOCITY AMPLITUDES AND EVOLUTION WITH THE RE NUMBER OF THE MAIN FREQUENCIES OF BOTH THE STRUCTURE AND THE WAKE.

As it can be observed, for $R e$ smaller than $4.5 \times 10^{5}$ amplitudes of the structure's vibration velocity remain constant and the main frequencies of both the structure and the wake agree and evolve linearly with $R e$, following a constant Strouhal number of $S t=0.20$. The mechanical resonance at $f_{2}$ can be decomposed in two phases. The first one corresponds to the $R e$ range $\left[4.5 \times 10^{5}\right.$ to $5.0 \times 10^{5}$ ], where the main structural frequency $f_{s}$ is equal to the natural frequency $f_{2}$, but differs from $\mathrm{f}_{\mathrm{w}}$. In this case, amplitudes of the structural vibration velocity increase slightly. This phase will be named pure mechanical resonance. The second phase corresponds to $R e$ ranging from $5.0 \times 10^{5}$ to $6.0 \times 10^{5}$ for which the excitation frequency $f_{w}$ becomes equal to the natural frequency $f_{2}$ and to the structural response frequency $f_{s}$. During this phase the amplitudes of the structural velocity increase strongly. This phase is known as lock-in and is characterized by a strong interaction between the mechanical displacement of the structure and the surrounding fluid flow. It is of prime interest to notice that the pure mechanical resonance appears earlier (ie: at smaller $R e$ numbers) than the lock-in, while the excitation frequency $f_{w}$ is still different from the natural frequency of the plate $f_{2}$. Also, it should be pointed out that the lock-in occurs at a Reduced Velocity value (RU=4.7) smaller than the expected value given by $\frac{1}{S t}=5$ and takes place up to $R U=5.6$. These considerations should be taken into account by designers when calculating the velocities at which resonance and high deformations of the structure will occur.

For a further analysis of the wake, three different Reynolds numbers will be studied in details. The first $\operatorname{Re}$ number of interest (A) is equal to $3.0 \times 10^{5}$ and corresponds to a flow velocity where there is no mechanical resonance and no lock-in. The second Re number of interest (B) is equal to $4.5 \times 10^{5}$ and corresponds to pure mechanical resonance without lock-in. The third Re number of interest (C) is equal to $5.4 \times 10^{5}$ and corresponds to the lock-in. Table 2 reports the characteristics of the 3 studied operating points. 


\begin{tabular}{|l|l|c|l|l|}
\hline & Reynolds & $\frac{\boldsymbol{U}_{\mathbf{0}}}{\boldsymbol{f}_{\mathbf{2}} \boldsymbol{D}}$ & Specification & Frequencies \\
\hline A & $\mathrm{Re}=3.0 \times 10^{5}$ & 2.77 & No resonance & $\mathrm{f}_{\mathrm{w}}=\mathrm{f}_{\mathrm{s}} \neq \mathrm{f}_{2}$ \\
\hline $\mathbf{B}$ & $\mathrm{Re}=4.5 \times 10^{5}$ & 4.17 & $\begin{array}{l}\text { No lock-in } \\
\text { Resonance }\end{array}$ & $\mathrm{f}_{\mathrm{w}} \neq \mathrm{f}_{\mathrm{s}}=\mathrm{f}_{2}$ \\
\hline C & $\mathrm{Re}=5.4 \times 10^{5}$ & 5.00 & $\begin{array}{l}\text { Lock-in and } \\
\text { resonance }\end{array}$ & $\mathrm{f}_{\mathrm{w}}=\mathrm{f}_{\mathrm{s}}=\mathrm{f}_{2}$ \\
\hline
\end{tabular}

TABLE 2: FLOW VELOCITY CONDITIONS OF THE STUDY FOR FURTHER ANALYSIS BY POD.

\subsection{Near wake structure}

Before analyzing in detail the individual structures of the near wake, it is worth having a look on the mean flow which provides the general characteristics of the wake. The vertical profiles of the mean stream-wise velocity $\mathrm{U}$, extracted at $\frac{x}{D}=$ 4 downstream the trailing edge, are reported in figure 9. The symmetry of the profile indicates that the mean flow of the wake is symmetric on both sides of the plate axis $(y=0)$ for these flow velocities. As expected, the thickness of the mean wake decreases when increasing the Re number from $3,0 \times 10^{5}$ to $5.4 \times 10^{5}$. The significant difference between the profiles is in the value of the maximum drift velocity of the wake $\frac{U_{d}}{U_{0}}=$ $\frac{U_{0}-U}{U_{0}}(\mathrm{y}=0)$ achieved on the symmetry axis. The momentum thickness $\theta=\int_{-\infty}^{+\infty} \frac{U}{U_{0}}\left(1-\frac{U}{U_{0}}\right) d y$ has been evaluated by integrating the velocity profiles. In the far wake, $2 \frac{\theta}{c}$ is representative of the drag coefficient. We observe a decrease of $\theta$ from $\operatorname{Re}=3 \times 10^{5}$ to $\mathrm{Re}=4.5 \times 10^{5}$, as usually expected from the evolution of the drag coefficient according to the Reynolds number for a steady plate. However, from $\mathrm{Re}=4.5 \times 10^{5}$ to $\operatorname{Re}=5.4 \times 10^{5}$, an increase in the normalized drift velocity $\frac{U_{d}}{U_{0}}$ as well as an increase in $\theta$ occur, which attest for an increase in the drag coefficient at mechanical resonance and lock-in.

The first modes are used to identify different kinds of vortices modes such as von Karman vortices, shear layer vortices and near wake bubble, as in the study of Miyanawala [12]. Modes of interest are those containing a high percentage of the total energy. For the purpose, an analysis of the distribution of modal energy has been achieved. It has been observed that the ten first modes contain $50 \%$ of the total energy and the 350 first modes contain $90 \%$ of the total energy (cf. Figure 10). For the following part of the analysis, only the first 5 modes will be studied because each mode higher than 5 contain less than $2 \%$ of the total energy. Table 3 reports the fraction of energy contained in each first 5 modes in percentage of the cumulated energy of these 5 modes. For each flow velocity, the highest energy is concentrated on mode number 1 and 2 which corresponds to the most energetic wake structures.
Total energy contribution of modes 1 and 2 approximates $40 \%$ of the total energy of the wake, and its contribution to the cumulated energy of the first 5 modes is higher than $80 \%$.



FIGURE 9: MEAN STREAMWISE VELOCITY PROFILES AT LOCATION $X=4 . D$ DOWNSTREAM OF THE TRAILING EDGE.


FIGURE 10: FRACTION OF TOTAL ENERGY OF EACH MODE AND CUMULATED ENERGY ACHIEVED BY COMBINING MODES.

The cartographies of the stream-wise velocity component of the first 5 POD modes obtained at the 3 different Reynolds numbers of interest are displayed in figures 9, 10 and 11 respectively. Based on these cartographies, it is possible to identify the different basic wake's structure. For the three studied Reynolds, the modes 1 and 2 correspond to the von Karman street because these modes are antisymmetric and correspond to alternating shedding of clock-wise and counterclockwise vortices. 


\begin{tabular}{|l|l|l|l|}
\hline & \multicolumn{3}{|l|}{$\begin{array}{l}\text { Energy fraction of the cumulated energy } \\
\text { of the 5 first modes }\end{array}$} \\
\hline Reynolds & $\mathbf{3 . 0} \times \mathbf{1 0}$ & $\mathbf{4 . 5} \times \mathbf{1 0}$ & $\mathbf{5 . 4} \times \mathbf{1 0}^{\mathbf{5}}$ \\
\hline Mode 1 & $42.66 \%$ & $42.92 \%$ & $43.37 \%$ \\
\hline Mode 2 & $41.04 \%$ & $42.29 \%$ & $42.83 \%$ \\
\hline Mode 3 & $5.65 \%$ & $7.00 \%$ & $4.80 \%$ \\
\hline Mode 4 & $5.56 \%$ & $3.93 \%$ & $4.63 \%$ \\
\hline Mode 5 & $5.09 \%$ & $3.86 \%$ & $4.37 \%$ \\
\hline
\end{tabular}

TABLE 3: ENERGY FRACTION OF THE FIRST 5 MODES, RATIO TO THE CUMULATED ENERGY.

At Reynolds $3 \times 10^{5}$, modes 3, 4 and 5 are identified as shear layer vortices modes. Shear layer vortices come from the boundary layer's unsteadiness. For the chord to thickness ratio of the plate considered here, the separated shear layer, over a stationary plate, is expected to reattach the plate's surface far upstream from the trailing edge and the separated shear layer would not influence strong fluctuations in the near wake [13]. Nevertheless, in case of a vibrating plate, the detachment of a leading edge vortex can contribute to the shear layer vortices.

At Reynolds $4.5 \times 10^{5}$, modes 4 is identified as a shear layer vortices mode. Mode 5 is considered as a combination of shear layer vortices and von Karman vortices modes. The bubble wake structure has the specificity of being symmetric, it is visible on mode 3 at $4.5 \times 10^{5}$. It extends up to $2 \mathrm{D}$ downstream the plate

At Reynolds $5.4 \times 10^{5}$, modes 3, 4 and 5 correspond also to the combination of shear layer vortices and von Karman vortices. Consequently, the main observation is that mode 3 shifts from a shear layer vortices mode to a bubble wake mode during the pure mechanical resonance and returns to a shear layer vortices mode during the lock-in. The bubble wake observed at $\operatorname{Re}=4.5 \times 10^{5}$ seems to be responsible for the decrease in the drift velocity of the near wake that was pointed out downstream the bubble wake.

Table 4 reports the energy contribution of the von Karman street, the shear layer and the bubble wake modes as a fraction of the cumulated energy of the 5 first modes. The percentage of energy present in the von Karman street increases linearly with flow velocity. It confirms that part of the shear layer vortices energy is transmitted to the near wake bubble energy. The von Karman street and the shear layer may act as an excitation source on the structure because of their nonsymmetrical specificity.

\subsection{Time response of the near wake modes}

The time response of the eigenvalues of the different POD modes has been analyzed. This enables to evaluate the main frequencies of each mode and to evaluate which mode acts as an excitation source on the structure.



FIGURE 11: CARTOGRAPHY OF $\Phi_{u}{ }^{i}(1 \leq i \leq 5)$ AT Re $=$ $3.0 \times 10^{5}$.


FIGURE 12: CARTOGRAPHY OF $\Phi_{u}{ }^{i}(1 \leq i \leq 5)$ AT RE = $4.5 \times 10^{5}$. 




FIGURE 13: CARTOGRAPHY OF $\Phi_{u}{ }^{i}(1 \leq i \leq 5)$ AT RE $=$ $5.4 \times 10^{5}$.

\begin{tabular}{|l|c|c|c|}
\hline & \multicolumn{3}{|c|}{$\begin{array}{l}\text { Energy percentage relative to the total } \\
\text { energy of the 5 first modes }\end{array}$} \\
\hline Reynolds & $\mathbf{R e}=\mathbf{3 . 0} \times \mathbf{1 0}^{\mathbf{5}}$ & $\mathbf{R e}=\mathbf{4 . 5} \times \mathbf{1 0}^{\mathbf{5}}$ & $\mathbf{R e}=\mathbf{5 . 4} \times \mathbf{1 0}^{\mathbf{5}}$ \\
\hline $\begin{array}{l}\text { Karman } \\
\text { street }\end{array}$ & $\begin{array}{c}83.7 \% \\
\text { Modes } 1+2\end{array}$ & $\begin{array}{c}85.2 \% \\
\text { Modes } 1+2\end{array}$ & $\begin{array}{c}86,2 \% \\
\text { Modes } 1+2\end{array}$ \\
\hline $\begin{array}{l}\text { Shear } \\
\text { layer }\end{array}$ & $\begin{array}{c}16.3 \% \\
\text { Modes } 3.4 .5\end{array}$ & $\begin{array}{c}7.0 \% \\
\text { Modes } 4.5\end{array}$ & $\begin{array}{c}13.8 \% \\
\text { Modes } 3.4 .5\end{array}$ \\
\hline $\begin{array}{l}\text { Bubble } \\
\text { wake }\end{array}$ & $0 \%$ & $\begin{array}{c}7.8 \% \\
\text { Mode } 3\end{array}$ & $0 \%$ \\
\hline
\end{tabular}

TABLE 4 FRACTION OF TOTAL ENERGY OF EACH TYPE OF WAKE STRUCTURE (FOR 5 FIRST MODES).

Figure 14 shows the power spectral density of mode 1 (ie von Karman street) for the 3 studied velocities. The characteristic frequencies $f_{v s}$ at which the maximum amplitude of the spectra is reached are respectively equal to 93,146 and $179 \mathrm{~Hz}$ at Reynolds $3.0 \times 10^{5}, 4.5 \times 10^{5}$ and $5.4 \times 10^{5}$ respectively, which gives a St number range between 0.186 and 0.199 . Here is a significant increase of the spectrum peak amplitude for the lock-in at Reynolds $5.4 .10^{5}$ and its frequency is very close to the natural frequency of the plate $f_{2}$. Interestingly, the power density spectrum of the von Karman street is a broad band spectrum at Reynolds $4.5 .10^{5}$ which corresponds to the mechanical resonance without lock-in.



FIGURE 14: POWER DENSITY SPECTRA OF KARMAN MODE (MODE 1) FOR Re $=3.0 \times 10^{5}, 4.5 \times 10^{5}$ AND 5.4 $\times 10^{5}$.



FIGURE 15: POWER DENSITY SPECTRA OF SHEAR LAYER MODE (MODE 4) FOR Re $=3.0 \times 10^{5}, 4.5 \times 10^{5}$ AND $5.4 \times 10^{5}$.



FIGURE 16: POWER DENSITY SPECTRUM OF BUBBLE WAKE MODE (MODE 3) AT Re $=4.5 \times 10^{5}$.

The power spectral density spectra of mode 4 characteristic of the shear layer vortices are displayed in figure 15 for the 3 Reynolds of interest. The spectra are broad-band spectra. Two main frequencies can be identified on the spectra, associated to a first peak at low frequency (f ranging between 7 $\mathrm{Hz}$ and $54 \mathrm{~Hz}$, frequency scaled by $\mathrm{D} / \mathrm{U}_{0}$ between 0.014 and 0.07 ) and a second peak at moderate frequency (f ranging between 63 and $135 \mathrm{~Hz}$, frequency scaled by $\mathrm{D} / \mathrm{U}_{0}$ between 0.13 and 0.15 ). Note that at $\mathrm{Re}=3 * 10^{5}$, far from resonance of the structure, the amplitudes of the main frequencies of the shear layer are not significantly higher than the average amplitude in the bandwidth of the spectrum. At $\mathrm{Re}=4.5 \times 10^{5}$, the spectrum of the shear layer vortices mode exhibits a single main frequency 
of $54 \mathrm{~Hz}$ (ie:0.07D/U0). For this particular Reynolds number, there is no overlap of the frequency range of mode 4 with the frequency range of the von Karman structures (mode 1). This justifies that, at $\mathrm{Re}=4.5 \times 10^{5}$, the shear layer vortices cannot exchange energy with the von Karman vortices and cannot lock the Karman vortices on the natural frequency of the structure (in agreement with Figure 8).

The power spectral density spectra of mode 3 measured at $\operatorname{Re}=4.5 \times 10^{5}$, characteristic of the bubble wake is shown in figure 16. The frequency of the main peak of the spectrum is equal to $9,8 \mathrm{~Hz}$. The spectrum is a narrow band spectrum containing energy for frequencies in the bandwidth [0$0.05 \mathrm{D} / \mathrm{U} 0]$. For this particular Reynolds number, there is an overlap of the frequency ranges of mode 4 and mode 3 which attests that shear layer vortices give energy rather to the nearwake bubble than to the von Karman vortices at pure mechanical resonance without lock-in.

\subsection{Von Karman street's vortices characterization}

An analysis of the characteristics of the vortices of the von Karman street has been realized for the three Reynolds of interest. Analysis is based on mode 1. The values of the mean circulation $\Gamma$, mean area, as well as the mean stream-wise distance between the vortices $L_{\text {vortex }}$ are summarized in table 5 . All these values are scaled using the upstream velocity $\mathrm{U}_{0}$ and the thickness of the plate D.



It is observed that the vortex area expands at the mechanical resonance and lock-in $\left(\mathrm{Re}=5.4 \times 10^{5}\right)$. The normalized circulation of the vortices tends to decrease with the Reynolds number. As a first approximation, $\frac{2 \Gamma}{U_{0} C}$ is representative of the rms value of the fluctuating lift coefficient, induced by the von Karman vortices. For a steady body, the evolution of this rms value with respect to the Reynolds number at high Reynolds numbers $\left(>10^{3}\right)$ is so not obvious, as evidenced by Norberg [14].

\section{CONCLUSION}

This study provides an experimental investigation of the structure-interactions of an elongated blunt plate at high Reynolds numbers based on POD analyses for the flow and modal analyses for the structure. Power spectral density analysis has highlighted that the main frequencies of both the PIV velocity field and the structural response are in agreement and evolve linearly with the flow velocity, at constant Strouhal number of 0.2 , except for Reynolds numbers ranging from $4.5 \times 10^{5}$ to $6.0 \times 10^{5}$. For this range, the mechanical resonance occurs at the natural frequency of the twisting mode. This mechanical resonance is characterized by high amplitude structural velocities which results in the lock-in phenomenon's occurrence at a narrow Reynolds number range between $5.1 \times 10^{5}$ to $6.0 \times 10^{5}$. The discrepancy between the Reynolds ranges of mechanical resonance and lock-in is of particular interest. The POD analysis has highlighted the fact that a near wake bubble is present for Reynolds equal to $4.5 \times 10^{5}$ and is induced by a transfer of the shear layer energy. For elongated blunt plates, further studies are necessary to characterize the dynamics of the shear layer. In particular, it is necessary to investigate the leading edge vortex dynamics at mechanical resonance and lock-in.

\section{ACKNOWLEDGEMENT}

This works is part of a common project between the LMSSC from CNAM/PARIS and IRENav from Ecole Navale/Arts et Métiers. It was partially granted by the CARNOT ARTS that is gratefully acknowledged. Many thanks to the technical support of IRENav and LMSSC.

\section{REFERENCES}

[1] Astolfi, J.-A., Lelong, A., Bot, P., Marchand, J.-B., "Experimental analysis of hydroelastic response of flexible hydrofoils, Proceedings of 5th High Performance Yacht Design Conference, Auckland, 10-12 March 2015.

[2] Ducarne, J., Modelisation et optimisation de dispositifs non-linéaires d'amortissement de structures par systèmes piézoélectriques commutés, PHD, CNAM, 27 March 2009.

[3] Williamson, C.H.K., Govardhan, R., (2008) A brief review of recent results in vortex-induced vibrations, Journal of Wind Engineering and Industrial Aerodynamics, 96, 713-735.

[4] G. S. Triantafyllou, M. Triantafyllou, M. Grosenbaugh, Optimal thrust development in oscillating foils with application to fish propulsion, Journal of Fluids and Structures 7 (2), 1993 205-224.

[5] J.Moubogha Moubogha, U. Ehrenstein, J.A. Astolfi, Forces on a pitching plate: An experimental and numeri cal study, Applied Ocean Research, Volume 69, 2017, Pages 2737,2017 https://doi.org/10.1016/j.apor.2017.09.003.

[6] Rostami, A.B., Mobasheramini, M. \& Fernandes, A.C. (2019) Strouhal number of flat and flapped plates at moderate 
Reynolds number and different angles of attack: experimental data. Acta. Mech. 230, 333-349.

[7] Chen, J. M., Fang, Y., (1996) Strouhal numbers of inclined flat plates, Journal of Wind Engineering and Industrial Aerodynamics, Vol. 61, Issues 2-3, 99-112.

[8] Shi, L.L., Liu, Y.Z., Yu, J. (2010) PIV measurement of separated flow over a blunt plate with different chord-tothickness ratios, Journal of Fluid and Structures, Vol. 26, 644657.

[9] Aaron Towne, A., Schmidt, O. T., Colonius T., (2018) Spectral proper orthogonal decomposition and its relationship to dynamic mode decomposition and resolvent analysis, Journal of Fluid Mechanics, Vol. 847, 821-867.

[10] Sirovich, L., (1987) Turbulence and the Dynamics of Coherent Structures. I- Coherent structures. II- Symmetries and Transformations. III- Dynamics and Scaling, Quart. Appl. Math., Vol. 45, 561-571.

[11] Graftieaux, L., Micherd, M., Grosjean, N., (2001) Combining PIV, POD and vortex identification algorithms for the study of unsteady turbulent swirling flows, Meas. Sci. Technol., Vol 12, 1422-1429.

[12] Miyanawala, T.P., Jaiman, R.K., (2018) Decomposition of wake dynamics in fluid-structure interaction via low-dimensional models, Journal of Fluid Mechanics, Vol. 867, 723-764.

[13] Toebes, G.H., Eagleson, P.S., (1961) Hydroelastic Vibrations of Flat Plates Related to Trailing Edge Geometry, Journal of Basic Engineering, December 1961, 671-678.

[14] Norberg, C., (2001) Flow around a circular cylinder: aspects of fluctuating lift, Journal of Fluid and Structures, Vol $15,459-46$ 